\title{
Lewis Causation Is a Special Case of Spohn Causation
}

\author{
Franz Huber \\ penultimate version: please cite the paper in the \\ British Journal for the Philosophy of Science
}

Lewis ([1973b]) famously defined event $c$ to be causally relevant to event $e$ in world $w$ just in case $O(c)$ and $O(e)$ as well as $\neg O(c) \square \rightarrow \neg O(e)$ are true in $w$, where $O(c)$ and $O(e)$ say that $c$ respectively $e$ occurs. For him this implied that $O(c) \square \mapsto O(e)$ is true, because he was assuming the underlying logic to be $\mathbf{V C}$, which validates $(\alpha \wedge \gamma) \supset(\alpha \square \mapsto \gamma)$. If one works with the weaker system $\mathbf{V}$ the causal relevance of event $c$ to event $e$ has to be defined as follows:

1. both $c$ and $e$ occur in $w$; i.e. $O(c)$ and $O(e)$ are true in $w$

2. if $c$ had not occurred, $e$ would not have occurred either in $w$; i.e. $\neg O(c) \square \mapsto$ $\neg O(e)$ is true in $w$

3. if $c$ had occurred, $e$ would have occurred as well in $w$; i.e. $O(c) \square \rightarrow O(e)$ is true in $w$

A typical semantics for counterfactual conditionals says that 'if $A$ were the case, $C$ would be the case', $\alpha \square \rightarrow \gamma$, is true if and only if $C$ is true in all the closest $A$-worlds (Lewis [1973a]; Stalnaker [1968]). Closeness or distance is spelt out in terms of a relation between possible worlds. In another paper (Huber [ms]) I have defended a ranktheoretic semantics for counterfactual conditionals based on a principle, called the Royal Rule, linking objective modalities and subjective beliefs. This principle implies that distance has the structure of a ranking function.

Ranking functions have been developed in (Spohn [1988]). They can be defined as functions $r$ from an algebra of propositions $\mathcal{A}_{W}$ over a set of possible worlds $W$ into the set of extended natural numbers $N \cup\{\infty\}$ such that the tautological proposition $W$ is assigned rank $0, r(W)=0$, the contradictory proposition 
$\emptyset$ is assigned rank $\infty, r(\emptyset)=\infty$, and the rank of a disjunction $A \cup B$ equals the minimum of the ranks of the disjuncts $A$ and $B, r(A \cup B)=\min \{r(A), r(B)\}$.

According to this ranktheoretic semantics a counterfactual conditional $\alpha \square \rightarrow$ $\gamma$ is true at world $w$ in model $\left(W, \mathcal{A}_{W},\left(r_{w}\right)_{w \in W}, \varphi\right)$ if and only if $\varphi(\alpha)^{r_{w}} \subseteq \varphi(\gamma)$. Here $W$ is the set of all possible worlds, $\mathcal{A}_{W}$ is an algebra of propositions over $W$, $\left(r_{w}\right)_{w \in W}$ is a family of ranking functions defined on $\mathcal{A}_{W}$, and $\varphi$ is an interpretation function on the underlying language $\mathcal{L} . \varphi(\alpha)$ is the set of worlds $w$ from $W$ in which the sentence $\alpha$ from the language $\mathcal{L}$ is true, and $\varphi(\alpha)^{r_{w}}$ is the set of $r_{w^{-}}$ minimal elements of $\varphi(\alpha)$-that is, the set of worlds in which $\alpha$ is true and that are closest to $w$ from the point of view of $r_{w}$.

The system $\mathbf{V}$ is sound and complete with respect to this ranktheoretic semantics. If the models $\left(W, \mathcal{A}_{W},\left(r_{w}\right)_{w \in W}, \varphi\right)$ are weakly respectively strongly centered in the sense that $r_{w}\left(\left\{w^{\prime}\right\}\right)=0$ if respectively if and only if $w=w^{1}$, then the system $\mathbf{V W}$ respectively $\mathbf{V C}$ is sound and complete with respect to the resulting weakly respectively strongly centered ranktheoretic semantics. V, VW, and VC are axiomatized in Lewis ([1973a], ch. 6).

In terms of the ranktheoretic semantics for counterfactuals the last two clauses of Lewis' definition of causal relevance translate into

$$
\begin{aligned}
& \text { 2. } r_{w}(O(e) \mid \overline{O(c)})>r_{w}(\overline{O(e)} \mid \overline{O(c)}), \\
& \text { 3. } r_{w}(\overline{O(e)} \mid O(c))>r_{w}(O(e) \mid O(c)),
\end{aligned}
$$

provided $r_{w}(\overline{O(c)})$ and $r_{w}(O(c))$ are finite. Causation itself is then defined as the transitive closure of causal relevance.

There are several well-known counterexamples to Lewis' counterfactual theory of causation, and Lewis has since refined his account (Lewis [1986], [2000]). The interested reader is referred to the collection of articles in (Collins \& Hall \& Paul [2004]). In a parallel effort people have tried to provide a probabilistic theory of causation, starting with (Suppes [1970]) up to the sophisticated work of (Spirtes \& Glymour \& Scheines [2000]). (Pearl [2009]) and (Woodward [2003]) pay hommage to both traditions, although the former leans more towards the probabilistic, and the latter more towards the counterfactual paradigm.

(Spohn [1983], [2006]) develops a theory of causation in the tradition of the probabilistic paradigm except that he is working with ranking functions instead of probability measures. He is able to elegantly deal with the problems besetting

\footnotetext{
${ }^{1}$ If the algebra $\mathcal{A}_{W}$ is not rich enough to contain all singleton propositions $\left\{w^{\prime}\right\}$, the formulation is more complicated.
} 
Lewis' account-most notably causation by overdetermination and causation by preemption-but the one big problem his account faces is that it is subjective.

More precisely, causal relevance-actually (Spohn [2006]) starts with the narrower notion of direct causation-and causation are only defined relative to an epistemically interpreted ranking function. Spohn is aware of this problem and has developed a way to partly "objectify" ranking functions by associating them with propositions (Spohn [1993]). However, his attempt at objectification is more complicated than successful. In particular, the crucial notions of direct causation and causation are only conditionally objectifiable.

Given a semantics of counterfactuals in terms of objective ranking functions, one is able to interpret Spohn's account of causation in terms of these objective ranking functions. It is then interesting to consider the relation between this objective version of Spohn's account of causation and the version of Lewis' account of causation one obtains from the ranktheoretic semantics for counterfactuals.

Ignoring some details-in particular, (Spohn [2006]) assumes that effects never predate their causes-we can paraphrase Spohn's definition of direct causation of event $e$ by event $c$ in world $w$ as follows: both $c$ and $e$ occur in $w$; i.e. $O(c)$ and $O(e)$ are true in $w$, and Spohn's Inequation holds:

$R a\left(\overline{O(e)} \mid O(c) \cap H_{w}\right)-R a\left(O(e) \mid O(c) \cap H_{w}\right)>R a\left(\overline{O(e)} \mid \overline{O(c)} \cap H_{w}\right)-R a\left(O(e) \mid \overline{O(c)} \cap H_{w}\right)$

Here $H_{w}$ is the complete history of world $w$ up to right before the effect $e$, but excluding the cause $c$ (Spohn's framework allows him to give a precise formulation of this clause). As before, causation itself is then defined as the transitive closure of direct causation.

For Spohn $R a$ is an ideally rational agent's prior ranking function. According to the ranktheoretic semantics counterfactuals receive truth values relative to presuppositions or contexts (Stalnaker [1970], [1998]). If we assume, as is plausible, that the relevant context for direct causation is the complete history up to right before the effect but excluding the cause, $H_{w}$, and if we further assume that the agent can know that $c$ causes $e$ only if she is certain of the relevant modalities $T_{w}$, i.e. $R a(\cdot)=R a\left(\cdot \mid T_{w}\right)$, it follows from the Royal Rule that $r_{w}(\cdot)=R a\left(\cdot \mid H_{w} \cap T_{w}\right)$.

Given these assumptions Lewis causation turns out to be a special case of Spohn causation, because causal relevance is a special case of direct causation. Suppose $c$ is causally relevant to $e$ in $w$ in the sense of Lewis. Then the left-hand side of Spohn's Inequation is positive and the right hand-side of it is negative. Thus causal relevance implies direct causation. The converse is not true, because Spohn's Inequation may hold if both sides are, say, positive, but the right-hand side is greater than the left-hand side. According to (Spohn [2006], sct. 5) this 
happens in cases of causal overdetermination and trumping.

Franz Huber

Formal Epistemology Research Group

Zukunftskolleg and Department of Philosophy

P. O. Box 216

University of Konstanz

Germany

E-mail: franz.huber@uni-konstanz.de

\section{Funding}

My research was supported by the German Research Foundation through its Emmy Noether program.

\section{Acknowledgements}

I am grateful to Wolfgang Spohn for helpful comments on an earlier version of this paper.

\section{References}

[1] Collins, J. \& Hall, N. \& Paul, L.A. [eds., 2004]: Causation and Counterfactuals. Cambridge, MA: MIT Press.

[2] Huber, F. [ms]: New Foundations for Counterfactuals.

[3] Lewis, D.K. [1973a]: Counterfactuals. Cambridge, MA: Harvard University Press.

[4] Lewis, D.K. [1973b]: Causation. Journal of Philosophy 70, 556-567.

[5] Lewis, D.K. [1986]: Postscripts to "Causation". In D. Lewis (1986), Philosophical Papers II. Oxford: Oxford University Press, 172-213.

[6] Lewis, D.K. [2000]: Causation as Influence. Journal of Philosophy 97, 182197. 
[7] Pearl, J. [2009]: Causality: Models, Reasoning, and Inference. 2nd ed. Cambridge: Cambridge University Press.

[8] Spirtes, P. \& Glymour, C. \& Scheines, R. [2000]: Causation, Prediction, and Search. 2nd ed. Cambridge, MA: MIT Press.

[9] Spohn W. [1983]: Eine Theorie der Kausalität. Unpublished Habilitation thesis. Munich: LMU Munich. 1983.

[10] Spohn, W. [1988]: Ordinal Conditional Functions: A Dynamic Theory of Epistemic States. In W.L. Harper \& B. Skyrms (eds.), Causation in Decision, Belief Change, and Statistics II. Dordrecht: Kluwer, 105-134.

[11] Spohn, W. [1993]: Causal Laws are Objectifications of Inductive Schemes. In J. Dubucs (ed.), Philosophy of Probability. Dordrecht: Kluwer, 223-252.

[12] Spohn, W. [2006]: Causation: An Alternative. British Journal for the Philosophy of Science 57, 93-119.

[13] Suppes, P. [1970]: A Probabilistic Theory of Causality. Amsterdam: NorthHolland Publishing Company.

[14] Stalnaker, R.C. [1968]: A Theory of Conditionals. In N. Rescher (ed.), Studies in Logical Theory. American Philosophical Quaterly. Monograph Series 2. Oxford: Blackwell, 98-112.

[15] Stalnaker, R.C. [1974]: Pragmatic Presuppositions. In M.K. Munitz \& P. Unger (eds.), Semantics and Philosophy. New York: New York University Press, 197-213.

[16] Stalnaker, R.C. [1998]: On the Representation of Context. Journal of Logic, Language, and Information 7, 3-19. Reprinted in R. Stalnaker (1999), Context and Content. Oxford: Clarendon Press, 96-113.

[17] Woodward, J. [2003]: Making Things Happen: A Theory of Causal Explanation. Oxford: Oxford University Press. 